\title{
Heparinización directa del paciente frente a heparinización del circuito extracorpóreo en hemodiálisis: estudio comparativo
}

\author{
Raquel Pelayo Alonso, María Eugenia Cuadrado Mantecón, Patricia Martínez Álvarez, Marina Rojo Tordable, \\ Emilio Ibarguren Rodríguez, Marta Reyero López
}

Diplomados Universitarios en Enfermería. Servicio de Nefrología. Hospital Universitario Marqués de Valdecilla. España

\section{Resumen}

Objetivo: Determinar si la forma de administración de la heparina durante el tratamiento con hemodiálisis influye en el estado de coagulación del sistema extracorpóreo.

Metodología: Estudio cuasiexperimental en 27 pacientes en Hemodiálisis crónica. Se establecen dos periodos de estudio. En el primero, la heparina prescrita se administra a través del acceso vascular y durante el segundo periodo, a través del botón arterial del sistema extracorpóreo.

Resultados: Se estudiaron 27 pacientes, siendo el $37 \%$ mujeres, y la edad media $68,63 \pm 17,44$. El $63 \%$ de los pacientes realizaba Hemodiafiltración on line postdilucional. No hubo diferencias estadísticamente significativas en cuanto a la dosis y parámetros de diálisis, el AV, el tratamiento anticoagulante ni la técnica de HD. En cuanto al estado de coagulación, si agrupamos por puntuación mayor o menor de 5, en el primer periodo, el porcentaje medio agrupado inferior a 5 puntos alcanza el $94,09 \%$ mientras que en el segundo periodo es de $97,1 \%$, lo que sí resulta estadísticamente significativo $(p=0,035)$.

Conclusiones: Según nuestro estudio, administrar la Heparina sódica en el circuito extracorpóreo mejora el estado final de coagulación del sistema, lo que supone una menor pérdida sanguínea para el paciente.

\begin{tabular}{|c|}
\hline Correspondencia: \\
Raquel Pelayo Alonso \\
Unidad de Hemodiálisis. Servicio de Nefrología \\
$1^{\text {a }}$ planta Pabellón 2 de Noviembre \\
Hospital Universitario Marqués de Valdecilla \\
Avda. Valdecilla, s/n. 39008 Santander \\
E-mail: sanesteban6@gmail.com
\end{tabular}

PALABRAS CLAVE

- HEMODIÁLISIS

- HEPARINA SÓDICA

- ANTICOAGULACIÓN

Patient direct heparinization versus extracorporeal circuit heparinization in hemodialysis: a comparative study

\begin{abstract}
Objective: Determine whether the mode of administration of heparin during hemodialysis treatment influences the coagulation status of the extracorporeal system.

Methods: Quasi-experimental study in 27 patients on chronic hemodialysis. Two study periods were established. In the former the prescribed heparin was administered through the vascular access, during the second period through the arterial button of the extracorporeal system.
\end{abstract}

Results: 27 patients were studied, being $37 \%$ female, and the mean age $68.63 \pm 17.44 .63 \%$ of patients were treated with postdilutional online hemodiafiltration. There were no statistically significant differences in dose and dialysis parameters, vascular access, anticoagulant therapy or hemodialysis technique. Regarding the coagulation status, being grouped by varying score 5 . In the first period, 5 points lower than average percentage was $94.09 \%$, while in the second period was $97.1 \%$, with significant differences $(p=$ 0.035). 
Conclusions: In our study, the administration of sodium heparin in the extracorporeal circuit improves the coagulation end state of the system, which means less blood loss for the patient.

\section{PALABRAS CLAVE}

- HEMODIALYSIS

- SODIUM HEPARIN

- ANTICOAGULATION

\section{Introducción}

En las técnicas de Hemodiálisis (HD) se hace circular la sangre del paciente por un circuito extracorpóreo lo que favorece la coagulación de la sangre ${ }^{1}$.

Para mantener el circuito extracorpóreo permeable debemos recurrir a fármacos anticoagulantes, siendo el más utilizado la heparina. 2,3,4

La anticoagulación en HD está dirigida a prevenir la trombosis del circuito sanguíneo buscando los mínimos riesgos posibles para el paciente. ${ }^{1-6}$

El objetivo es utilizar la mínima dosis posible de heparina con la que el dializador y las líneas extracorpóreas queden limpios de restos hemáticos, minimizando así la pérdida hemática y los efectos adversos que sufren los pacientes por la heparinización repetida a lo largo del tiempo ${ }^{7}$.

Hay que tener en cuenta, que una anticoagulación insuficiente produce pérdidas hemáticas y de hierro, y condiciona una disminución del rendimiento depurativo de la diálisis. En general, la dosis que se emplea suele ser menor que la necesaria para la anticoagulación total del paciente, si bien, la necesidad de heparina es muy variable de unos pacientes a otros, dependiendo de factores propios del paciente y de la técnica de HD5 .

La heparina sódica (HS) ha sido, hasta el momento, el anticoagulante de primera elección, aunque su uso está siendo reemplazado por el empleo de la heparina de bajo peso molecular (HBPM) ${ }^{8}$.

Las guías de práctica clínica recomiendan el uso de HBPM frente a $\mathrm{HS}^{9}$, pero en la práctica no existe consenso sobre el empleo de un tipo u otro de heparina, necesidad de cebado con/sin heparina, dosis, pauta según modalidad de HD o modo de administrar la heparina $1,2,4,6,10,11$.
Algunos estudios apuntan a que la heparina administrada directamente al paciente proporciona un mayor efecto anticoagulante sobre el circuito extracorpóreo ${ }^{12,13}$. Es por ello, por lo que nos planteamos al presente estudio.

\section{Objetivo}

Determinar si la forma de administración de la HS (en el acceso vascular o en la línea arterial) durante el tratamiento con hemodiálisis influye en el estado de coagulación del sistema extracorpóreo.

\section{Pacientes y método}

Se realizó un estudio cuasiexperimental en 27 pacientes sometidos a HD crónica, en donde se aplicaron 2 métodos de administración de HS, durante 2 periodos de estudio de 5 semanas de duración cada uno de ellos. En ambos periodos, la dosis de HS prescrita para cada paciente fue la misma.

Durante el primer periodo, la HS se administraba a través del acceso vascular ( $A V$ ) del paciente, bien en el ramal venoso del CVC o a través de la aguja venosa. Tras la administración de la heparina, se realizaba un lavado con 10cc de suero salino para garantizar que la heparina pasase al torrente sanguíneo del paciente. Se comenzaba la sesión de HD transcurridos 3 minutos desde la administración de la heparina.

Durante el segundo periodo, la heparina se administraba a través del botón arterial del sistema extracorpóreo, una vez comenzada la sesión, cuando la sangre hubiera alcanzado el botón arterial, antes que la sangre llegara al dializador.

Los criterios de inclusión fueron ser pacientes en tratamiento con HD cuya heparinización se realizaba con HS y que consintieron ser incluidos en el estudio tras recibir la información pertinente. Se excluyeron a los pacientes que realizaban HD con restricción de heparina y en tratamiento con HBPM.

Como variables de estudio, de la Historia clínica del paciente, se recogieron la edad, el sexo, el AV, la nefropatía, la técnica de HD y si el paciente estaba en tratamiento domiciliario con antiagregantes 0 anticoagulantes. En cada sesión, se recogieron datos propios de la HD como son el flujo sanguíneo, presión venosa y presión arterial, tiempo de hemostasia en pacientes portadores de FAVI y estado de coagulación del siste- 
ma tras la sesión. Para valorar este dato, se diseñó una escala tipo Likert de 0 (sistema totalmente limpio) a 10 (sistema totalmente coagulado, no permitiendo el retorno sanguíneo). Además, cada semana, se determinó la eficacia dialítica (Kt/V) y se realizó un estudio hematológico (hematocrito, hemoglobina, actividad de protombina e INR).

Los datos recogidos fueron analizados mediante el paquete estadístico SPSS en su versión 15.0 para Windows, empleándose los test de chi cuadrado para las variables cualitativas y la prueba de los rangos de Wilcoxon para las variables cualitativas. Los resultados se consideraron significativos si el nivel crítico observado era inferior al $5 \%(p<0.005)$.

\section{Resultados}

Se estudiaron 27 pacientes, siendo el $37 \%$ mujeres, y la edad media $68,63 \pm 17,44$. La nefropatía más frecuente fue la de origen vascular, con un $40,7 \%$ de los casos. EI $63 \%$ de los pacientes era portador de FAVI y la técnica de HD más utilizada fue la hemodiafiltración (HDF) on line post-dilucional con un $63 \%$. El $66,7 \%$ de los pacientes no tenía prescrito tratamiento domiciliario con anticoagulantes 0 antiagregantes.

El número de sesiones analizadas fueron 399 durante el primer periodo y 372 en el segundo. No hubo diferencias estadísticamente significativas en cuanto a la dosis y parámetros de diálisis (Tabla 1).

Tabla 1. Parámetros y dosis de diálisis evaluadas durante ambos periodos de estudio

\begin{tabular}{|lccc}
\hline Parámetro & Periodo 1 & Periodo 2 & $\begin{array}{c}\text { Significación } \\
\text { estadística }(\mathbf{p})\end{array}$ \\
\hline Flujo de sangre $(\mathrm{ml} / \mathrm{min})$ & $325,52 \pm 31,38$ & $324,89 \pm 35,76$ & 0,849 \\
\hline Presión Venosa $(\mathrm{mmHg})$ & $167,15 \pm 26,97$ & $163,52 \pm 27,06$ & 0,292 \\
\hline Presión Arterial $(\mathbf{m m H g})$ & $-192,96 \pm 33,24$ & $-188,15 \pm 37,86$ & 0,163 \\
\hline $\begin{array}{l}\text { Tiempo de hemostasia } \\
\text { (minutos) }\end{array}$ & $8,65 \pm 1,16$ & $8,46 \pm 1,92$ & 0,68 \\
\hline $\begin{array}{l}\text { Sesiones con cambio de sistema } \\
\text { (número) }\end{array}$ & $0,07 \pm 0,385$ & $0,037 \pm 0,19$ & 0,66 \\
\hline Kt/V (ml/min/1.73m $\left.{ }^{2}\right)$ & $1.67 \pm 0,43$ & $1,68 \pm 0,49$ & 0,814 \\
\hline Puntuación Escala Coagulación & $1,59 \pm 1,12$ & $1,28 \pm 0,81$ & 0,08 \\
\hline
\end{tabular}

Los datos se expresan como la media \pm desviación típica.

Periodo 1: administración de heparina en acceso vascular. Periodo 2: administración

de heparina en botón arterial.

os datos se expresan como la media \pm desviación típica.
En el perfil hematológico (Tabla 2) sólo hubo diferencias en el nivel de plaquetas, siendo más bajo durante el primer periodo $(193,07 \pm 62,42$ vs $202,05 \pm 65,74$, $p=0,05$ ).

Tabla 2. Perfil hematológico de la muestra durante ambos periodos de estudio.

\begin{tabular}{lccc} 
Parámetro & Periodo 1 & Periodo 2 & $\begin{array}{c}\text { Significación } \\
\text { estadística }(\mathbf{p})\end{array}$ \\
\hline Hematocrito (\%) & $36,13 \pm 4$ & $35,6 \pm 4$ & 0,38 \\
\hline Hemoglobina (mg/dl) & $11,84 \pm 1,25$ & $11,82 \pm 1,17$ & 0,89 \\
\hline Plaquetas $\left(\times 10^{3} / \mu \mathrm{L}\right)$ & $193,07 \pm 62,42$ & $202,05 \pm 65,74$ & 0,05 \\
\hline Protrombina (\%) & $96,54 \pm 4,49$ & $97,22 \pm 4,05$ & 0,17 \\
INR & $0,98 \pm 0,07$ & $1 \pm 0,14$ & 0,40
\end{tabular}

Periodo 1: administración de heparina en acceso vascular. Periodo 2: administración de heparina en botón arterial.

En cuanto al estado de coagulación, la puntuación media durante el primer periodo fue de $1,59 \pm 1,12$ puntos frente a $1,28 \pm 0,81$ en el segundo $(p=0,08)$ (Figura 1$)$.

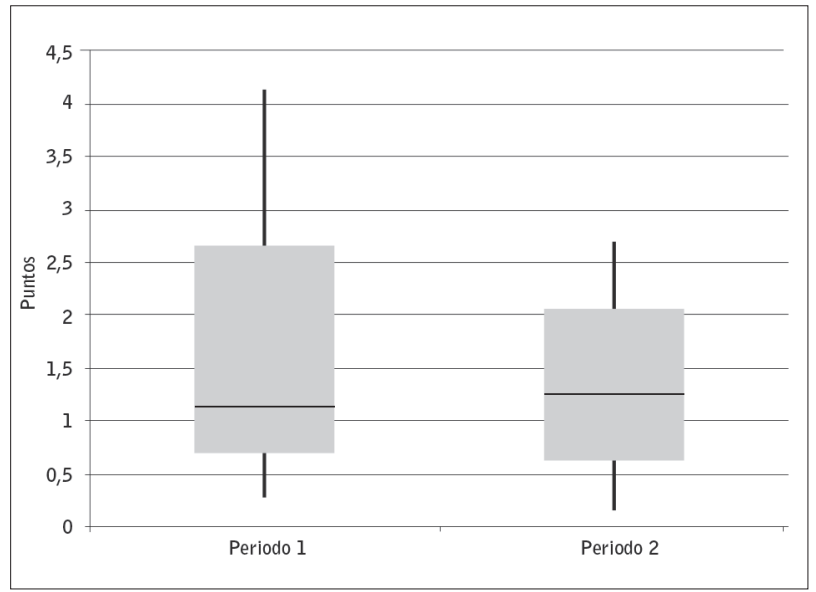

Figura 1. Puntuación de la escala de evaluación del estado de coagulación del sistema, según el periodo de estudio. (Periodo 1: administración de heparina en acceso vascular. Periodo 2: administración de heparina en botón arterial).

Si agrupamos por puntuación mayor o menor de 5, en el primer periodo, el porcentaje medio agrupado inferior a 5 puntos alcanza el $94,09 \%$ mientras que en el segundo periodo es de $97,1 \%$, lo que sí resulta estadísticamente significativo $(p=0,035)$ (Figura 2). 


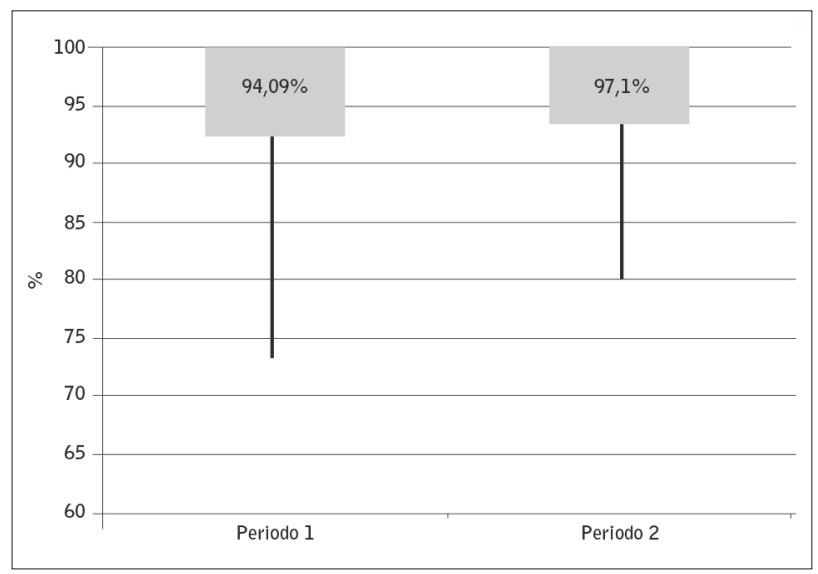

Figura 1. Porcentaje de pacientes que presentaron una puntuación $<5$ en la escala de evaluación del estado de coagulación del sistema según el periodo de estudio. (Periodo 1: administración de heparina en acceso vascular. Periodo 2: administración de heparina en botón arterial).

No influyeron estadísticamente, ni el AV, ni el tratamiento antiagregante, ni la técnica de HD.

\section{Discusión}

Pese a las recomendaciones de las guías clínicas ${ }^{9}$ no existen criterios universales de actuación, y cada centro actúa según sus propios criterios, existiendo una gran variabilidad clínica desde la necesidad o no de purgado del sistema, uso de un tipo u otro de heparina o incluso cómo administrar dicha heparina ${ }^{1}$.

La pauta de heparinización más frecuentemente utilizada, en un $92,6 \%$ de los pacientes sometidos a HD, consiste en la administración de un bolo inicial de heparina seguido de una pauta intermitente ${ }^{14}$. La duda que puede plantearse es dónde administrar dicho bolo inicial. En las técnicas de depuración continua, el método más utilizado es la administración de la heparina en la línea arterial del circuito ${ }^{15}$ mientras que en el caso de los sistemas de hemodiálisis, no existen datos concluyentes ${ }^{14}$.

Cuando se utiliza HBPM, debido a que las pérdidas de ésta por el dializador son mayores cuando se administra por la rama arterial del circuito, algún autor recomienda que en el caso de HD de alto flujo y especialmente en técnicas de HDF, la HBPM se administre a través de la vía venosa, para evitar el paso por el dializador ${ }^{16}$, o bien, se administre 3-4 minutos antes del comienzo de la sesión ${ }^{17}$.

Hay que tener en cuenta que estas recomendaciones se basan en el uso de HBPM. En cambio, no se hace referencia a la HS. Pese a que su peso molecular es discre- tamente mayor (unos $12000 \mathrm{Da}$ frente a los $4500 \mathrm{Da}$ de las HBPM, por término medio) se puede esperar un comportamiento similar, ya que las membranas y las técnicas de HD utilizadas en la actualidad permiten una eliminación eficaz de sustancias de un peso molecular similar a la $\mathrm{HS}^{18}$.

Cabrera LC y cols ${ }^{12,13}$ objetivan una menor necesidad de heparina cuando se administra a través del AV del paciente mientras que en nuestro estudio, obtenemos mejores resultados cuando la heparina se administra a través del circuito extracorpóreo. Ambos estudios siguen el mismo diseño metodológico pero con diferentes heparinas, HBPM frente a HS respectivamente.

El Estudio Español sobre anticoagulación en hemodiáli$\mathrm{sis}^{14}$, con una muestra de 6093 pacientes, determina que el $58,5 \%$ de los pacientes en HD está sometido a tratamiento anticoagulante 0 antiagregante. Sin embargo, en nuestra muestra, sólo el $33 \%$ de los pacientes tiene pautado este tratamiento. Esta diferencia puede radicar en que en nuestro estudio se excluyeron los pacientes en tratamiento con HBPM, que en nuestro centro, son pacientes con una mayor tasa de problemas de coagulabilidad, y por lo tanto, más proclives a estar en tratamiento con anticoagulantes 0 antiagregantes.

Debido a la gran variabilidad del efecto de la heparina de un paciente a otro, es preciso individualizar dicha dosis. Existen pruebas de laboratorio que permiten adecuar la anticoagulación, pero, en la práctica habitual, son poco utilizados, realizándose el ajuste de dosis por tanteo ${ }^{14}$, teniendo en cuenta criterios como el peso del paciente y especialmente, la valoración, mediante inspección visual, del estado de coagulación del sistema, método muy subjetivo y que supone una enorme variabilidad. Pese a ser un criterio muy usado, es un aspecto muy poco estudiado ${ }^{1}$.

En conclusión, los resultados de nuestro estudio objetivan que administrar la HS a través del circuito extracorpóreo mejora el estado final de coagulación del sistema, lo que condiciona una menor pérdida sanguínea para el paciente.

Recibido: 9 diciembre 2014

Revisado: 20 diciembre 2014

Modificado: 4 enero 2015

Aceptado: 5 enero 2015 


\section{Bibliografía}

1. Moreno JA, Pórtoles $\mathrm{JM}^{\mathrm{a}}$, Ochando A, Ortigosa A, Garrido $M^{a} V$. Estudio observacional prospectivo de los parámetros clínicos de la anticoagulación en el circuito extracorpóreo en una cohorte de pacientes en programa de hemodiálisis crónica. Comunicaciones presentadas al XXX Congreso de la Sociedad Española de Enfermería Nefrológica. [Consultado el 18 de febrero de 2014.] Disponible en: http://www.revistaseden.org/files/ 2849_135-140.pdf.

2. Belchí F, Alfaro A. Hemodiálisis sin heparina. Sistema de lavado continuo doble: ¿Mejora el sistema de lavado continuo prefiltro? Rev Soc Esp Enferm Nefrol 2006; 9(2): 132-135.

3. Davenport A. Optimization of heparin anticogulation for hemodialysis. Hemodial Int. 201; 15 Suppl (1): S43-8.

4. Del Valle MJ, Ardela J, Gómez V, Aranguren P. Necesidades de heparina en hemodiálisis. Influencia del purgado, la dosis inicial y el flujo sanguíneo. Comunicaciones presentadas al XVIII Congreso de la Sociedad Española de Enfermería Nefrológica. [Consultado el 18 de febrero de 2014.] Disponible en: http://www.seden.org/publicaciones_articulodet.asp?idioma $=E S \& i d a r t i c u l 0=1269$.

5. Fort J, Piera L. Anticoagulación y fribrinolisis. En: Jofre R, López Gómez JM, Pérez R, Rodríguez P (eds.). Tratado de hemodiálisis. $2^{\mathrm{a}}$ edición. BarceIona: Editorial Médica Jims; 2006. p.201-211.

6. Guéry B, Alberti $C$, Servais A, Harrami E, Bererjo $L$, Zins $B$ et al. Hemodialisys without systemic anticoagulation: A prospective randomized trial to evaluate 3 strategies in patients at risl of bleeding. Plos0ne 2014; 9(5):e97187 [PubMed].

7. Gaínza FJ. Problemas de coagulación de los circuitos extracorpóreos y estrategias para mejorar la duración de los filtros. Nefrología 2007; 27(Suppl 3):166-177.

8. Davenport A. What are the anticoagulation options for intermittent hemodialysis? Nat Rev Nephrol 2011 Jul; 7(9):499-508.

9. European Best Practice Guidelines Expert Group on Hemodialysis, European Renal Association: Section V. Chronic intermittent haemodialysis and prevention of clotting in the extracorporeal system. Nephrol Dial Transplant 2002; 17 (suppl 7): 63-71.
10. Sánchez I, Estupiñán S, Ledesma D, García de la Cruz N, Vera L, Ramírez IC et al. ¿Es necesario heparinizar el suero de cebado del dializador? Rev Soc Esp Enferm Nefrol 2011; 14(4): 215-221.

11. Ronda MJ, Crehuet I, Méndez P, Pulido JF. Conexión del paciente al monitor, En: Crespo R, Casas $\mathrm{R}$ (eds.).Procedimientos y protocolos con competencias específicas para Enfermería Nefrológica. $1^{a}$ edición, Madrid: Editorial Aula Médica;2013. pp.57-59.

12. Cabrera LC, Martín M, Gómez AS, Pizarro JL. Heparinización a través del acceso vascular. Enferm Nefrol 2013; 16 Suppl (1): 75-76.

13. Cabrera LC, Martín M, Gómez AS, Pizarro JL. Heparinización directa al paciente versus heparinización del circuito extracorpóreo. Enferm Nefrol 2012; Suppl (1):55.

14. Herrero JA, González E, Pérez R, Tornero F. Estudio español sobre anticoagulación en hemodiálisis. Nefrología 2012; 32(2): 143-152.

15. Santana L, Sánchez M, Ramírez A, Villanueva A. Depuración extrarrenal continua sin anticoagulación. Nefrología 2009; 29(6):608-609.

16. Davenport A. Low molecular weight heparin for routine hemodialysis. Hemodial Int 2008;12:53437.

17. Sabry A, Taha M, Nadal M et al. Anticoagulation therapy during haemodialysis: a comparative study between two heparin regimens. Blood Coagul Fibrinolysis 2009; 20:57-62.

18. Suranyi M, Chow J. Review: Anticoagulation for haemodialysis. Nephrology (Carlton) 2010;15:38692. 